\title{
Cardiovascular magnetic resonance imaging - a pictorial review
}

\section{Vijay Dahya, MB BCh, FCRad (SA) \\ Tuft and Partners Inc., and Department of Radiology, University of Cape Town \\ Bruce S Spottiswoode, PhD (Med) \\ MRC/UCT Medical Imaging Research Unit, Department of Human Biology, University of Cape Town; and Department of Radiological Sciences and Oncology, Stellenbosch University}

\section{Introduction}

Cardiovascular magnetic resonance imaging (CMR) is a powerful problem-solving tool and arguably offers the most comprehensive assessment of cardiac morphology and function, as well as the opportunity of rebuilding the bridge between cardiologists and radiologists. The role of CMR-trained imaging physicists is also valuable, and many CMR centres harmoniously incorporate these three sub-specialty fields.

This paper comprises an overview of several CMR techniques, outlining both the strengths and limitations of the modality.

\section{Cardiovascular magnetic resonance imaging}

The combinations of cardiac and respiratory motion make the heart a challenging organ to image. Electrocardiogram (ECG) gating is done almost without exception, and patients are often asked to hold their breath for each scanning sequence to minimise respiratory motion artefacts. In prospective gating, the acquisition is triggered by the QRS complex, and usually only $90 \%$ of the cardiac cycle is acquired. In retrospective gating, data are acquired continuously while the ECG trace is recorded, and processing is done retrospectively to provide images throughout the cardiac cycle.

MRI is a relatively slow modality, and samples need to be taken from a number of heartbeats. Image acquisition is made more time-efficient by capturing interleaved samples or slices during each heartbeat. The resulting samples from different phases of the cardiac cycle can be represented as a multi-slice collection of images, or interleaved into a cinematographical series of images.

Where patients are unable to hold their breath, respiratory gating can also be incorporated, either using a belt around the abdomen or
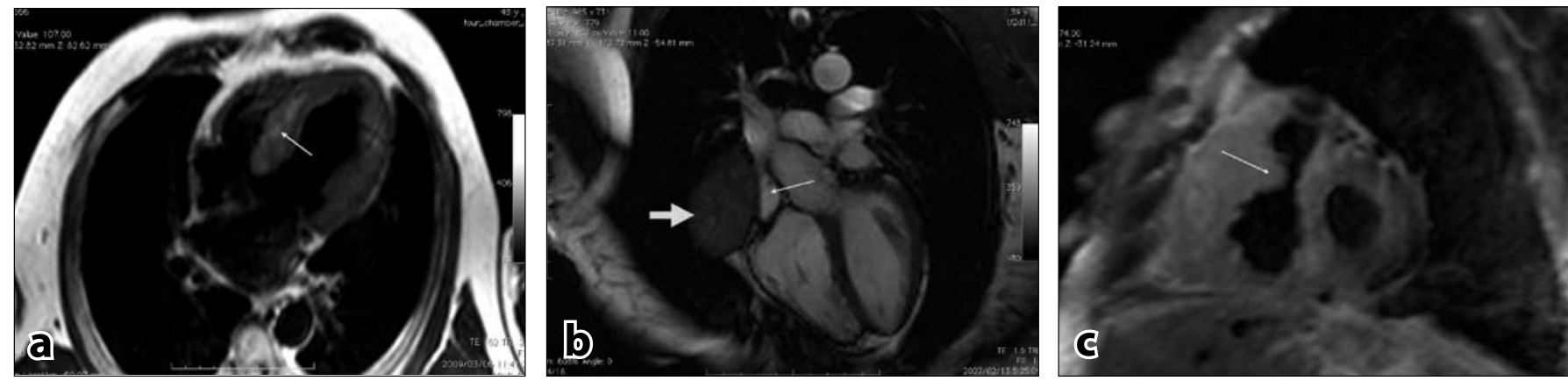

Fig. 1a. Four-chamber T2-weighted image showing evidence of active inflammation (arrow) in a patient with myocarditis. (1b.) Four-chamber bright-blood image showing a mediastinal neoplasm, post-operatively confirmed to be a benign thymoma (block arrow) impressing the right atrium (arrow) but not invading the heart. (1c.) Short axis post-contrast T1 image showing diffuse RV infiltration, confirmed on catheter directed myocardial biopsy to represent primary cardiac lymphoma, bulging into and severely narrowing the RV outflow tract (arrow).
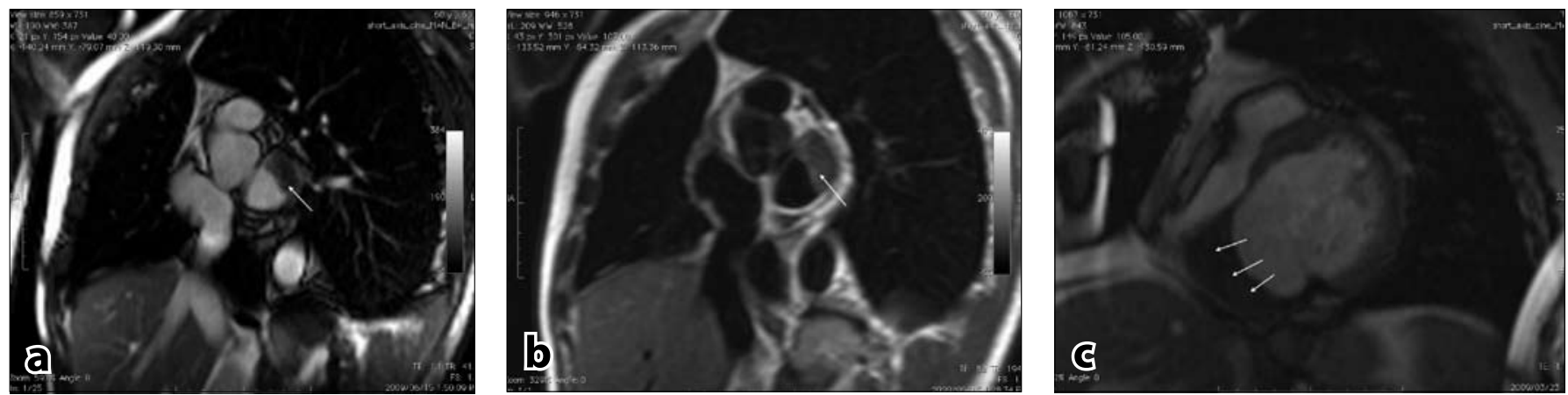

Fig. 2a. Bright-blood image showing thrombus (arrow) in a typical site with the left atrium. (2b.) Dark-blood image in the same patient as in (a) confirming thrombus (arrow) within the left atrium. CMR was used in this case following equivocal echocardiography for confirmation of thrombus and to differentiate this from an atrial myxoma. (2c.) Extensive mural thrombus (arrows) is shown in a patient with previous infero-septal myocardial infarction with aneurysm formation. 


\section{REVIEW ARTICLE}
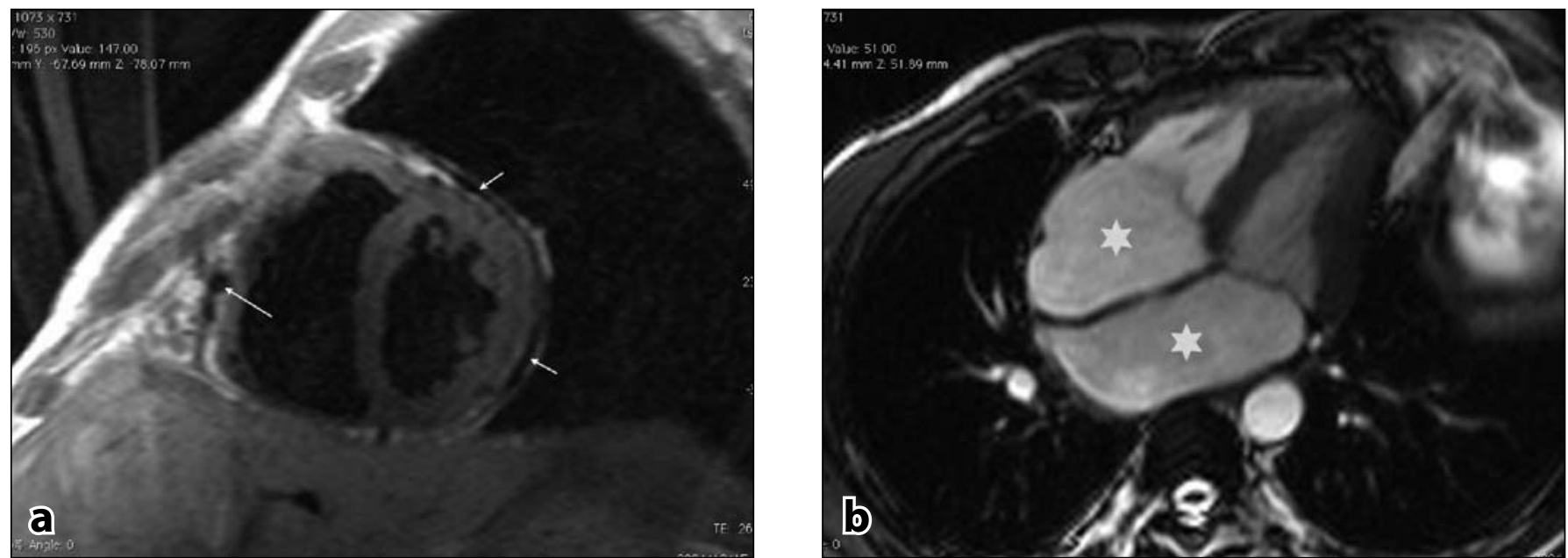

Fig. 3. Constrictive pericarditis. (3a.) Dark blood T1-weighted image clearly depicting the thickened pericardium (arrows) which is nodular in parts (long arrow). (3b.) Bright blood four-chamber image in the same patient showing bi-atrial enlargement (stars), a feature common to constrictive pericarditis and restrictive cardiomyopathy.

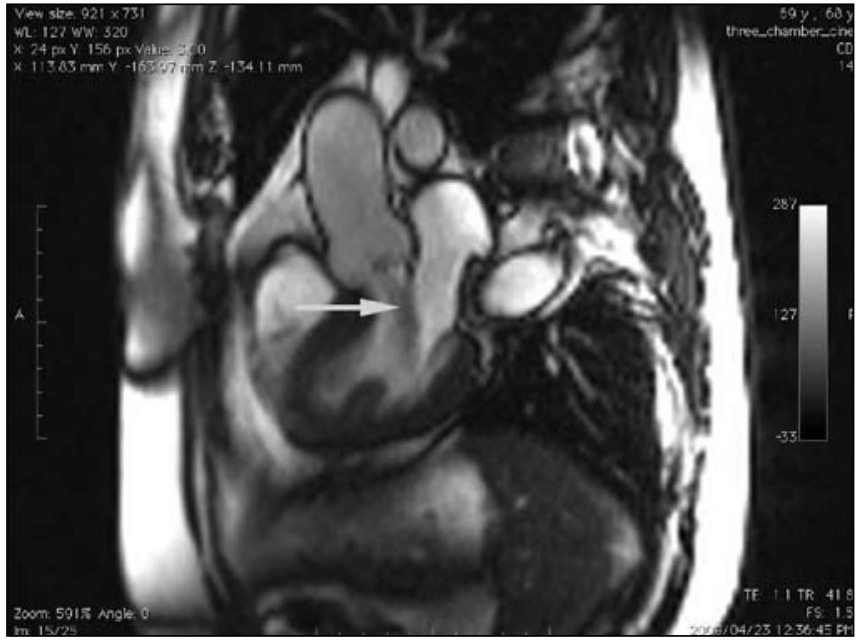

Fig. 4. Gradient echo bright blood image in the plane of the left ventricular outflow showing prominent low signal (spin dephasing) owing to turbulent flow from aortic regurgitation (arrow), leading to early closure of the mitral valve anterior leaflet.

monitoring the liver-lung interface using navigator echoes, which are incorporated in some MRI pulse sequences.

A number of fast imaging techniques have been developed, including steady state free precession (SSFP) and echo planar imaging (EPI). Details of these sequences can be found in many MRI texts. ${ }^{1}$ A specialised phased-array radiofrequency chest coil is used for cardiac imaging to maximise the MRI signal. If multiple phased-array coils are used, parallel imaging ${ }^{2,3}$ can be applied to significantly reduce the scan time, with only a marginal trade-off in signal.

\section{Techniques and clinical applications}

\section{Morphology}

Various image sequences can be used, either rendering 'dark blood imaging' where the blood pool is darker than the myocardium, or 'bright blood imaging' where the myocardium is darker than the blood pool. Cardiac imaging planes (short axis, long axis and four-chamber orientations) are traditionally used. Standard imaging planes (axial, coronal and sagittal) are usually also employed to ensure that the extracardiac structures are assessed.

CMR is used with excellent effect in patients with suspected cardiomyopathies, myocardial inflammation, tumours and infiltration (Fig. 1). Both the right ventricle (RV) and left ventricle (LV) can be viewed in their entirety.

Intracardiac thrombus can also definitively be identified and differentiated from neoplasms (e.g. atrial myxoma) with CMR as a problem-solving tool following equivocal echocardiography (Fig. 2).

Myocardial iron deposits associated with transfusion-dependent anaemias can be quantified using $\mathrm{T}^{\star}$ mapping. This allows pre-clinical monitoring of iron deposition with immense therapeutic implication (iron deposition can be reversed), as the first clinical manifestation is often intractable heart failure. ${ }^{4}$

CMR also offers the most complete evaluation of the pericardium. This allows the distinction between constrictive pericarditis and restrictive cardiomyopathy, which can both be clinically and haemodymically indistinguishable (Fig. 3).

General chamber orientation and morphology can be evaluated as well as abnormal communications (atrial septal defect, ventricular septal defect and patent ductus arteriosus), often applied to congenital cardiac disease.

\section{Function}

Both the right and left ventricular parameters can be evaluated with excellent reproducibility. A series of semi-automatically defined contours are used to obtain estimates of ventricular volume and myocardial mass. Evaluation of RV function is important in patients with suspected arrhythmogenic right ventricular cardiomyopathy (ARVC) and in therapeutic follow-up of patients after repair of certain congenital heart diseases, and cannot be reliably measured on any other non-invasive modality.

Stress tests can be performed where controlled doses of dobutamine can be administered to investigate wall motion abnormalities under 


\section{REVIEW ARTICLE}
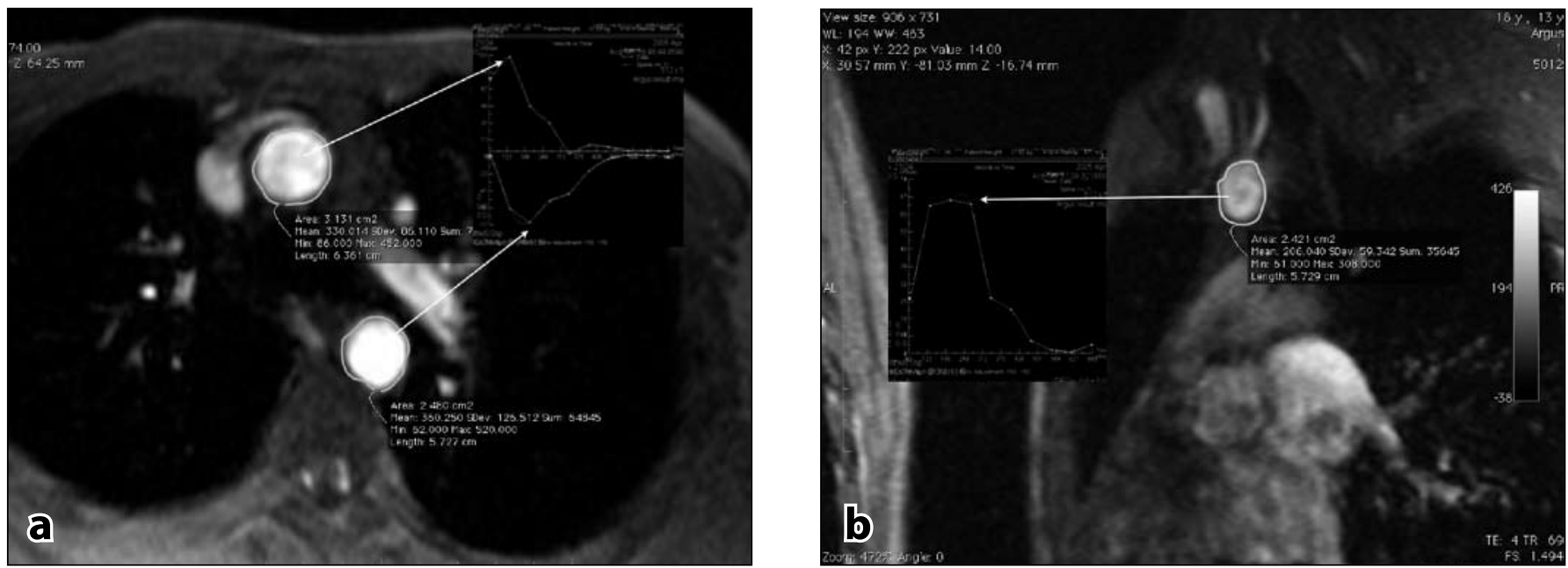

Fig. 5. A patent post aortic coarctation repair for evaluation of a residual stenosis at the repair site. (5a.) Regions of interest depicting the ascending and descending aorta on the axially orientated magnitude image with resultant in-laid velocity-time curves measured in the ascending and descending aorta respectively. (5b.) Region of interest through the repaired segment on the sagitally orientated magnitude image with in-laid resultant velocity-time curve through the repaired segment confirming no significant step-up in velocity and therefore no pressure gradient, consistent with successful repair.

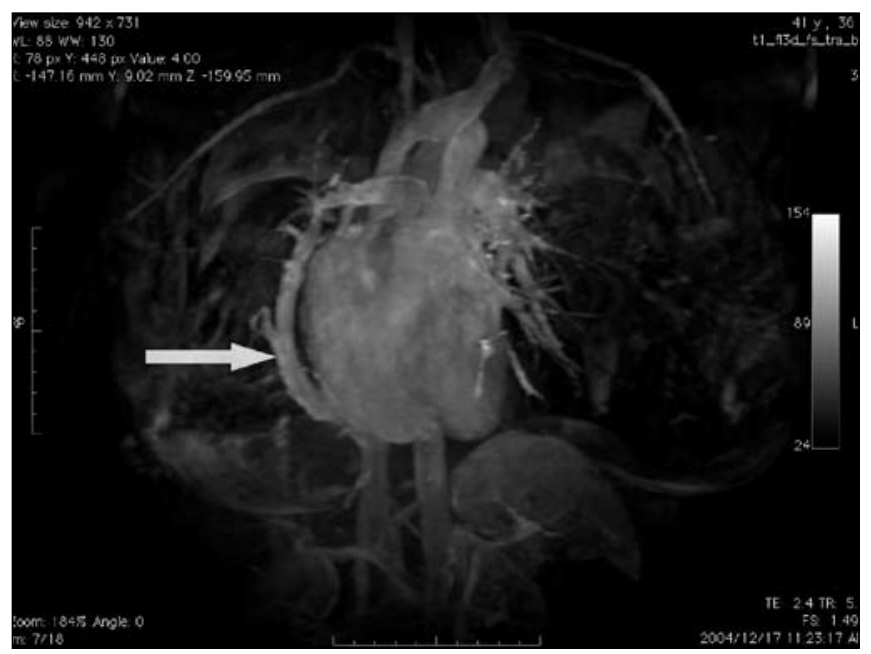

Fig. 6. Contrast-enhanced MR angiogram of a patient with Scimitar syndrome. Note the anomalous drainage of the right inferior pulmonary vein into the supra-hepatic IVC via the scimitar vein (block arrow). stress conditions. This is, however, less accurate than perfusion stress CMR (see Perfusion below).

Gradient echo cine images are also useful for valvular imaging and have a niche application since slow moving or turbulent blood results in low signal intensity (Fig. 4). These can be quantified with phase contrast (PC) velocity encoding, which provides an instantaneous measure of fluid velocity, and can be used to provide velocity-time and flow-time curves through the valves and the great vessels, thus allowing calculation of pressure gradients. Flow can also be measured in structures not easily or accurately assessed by echocardiography e.g. post-valvar pulmonary stenosis and aortic coarctation (Fig. 5). This technique can also be applied to the myocardium, yielding regional measures of velocity and strain rate. ${ }^{5,6}$ Three-dimensional (3D) phase contrast velocity encoding is also being used as an emerging technique for creating high-resolution angiograms without the need for contrast agents. ${ }^{7,8}$

\section{MR angiography}

3-D contrast angiography can be performed in conjunction with CMR to fully evaluate the great vessels, and is particularly useful in patients
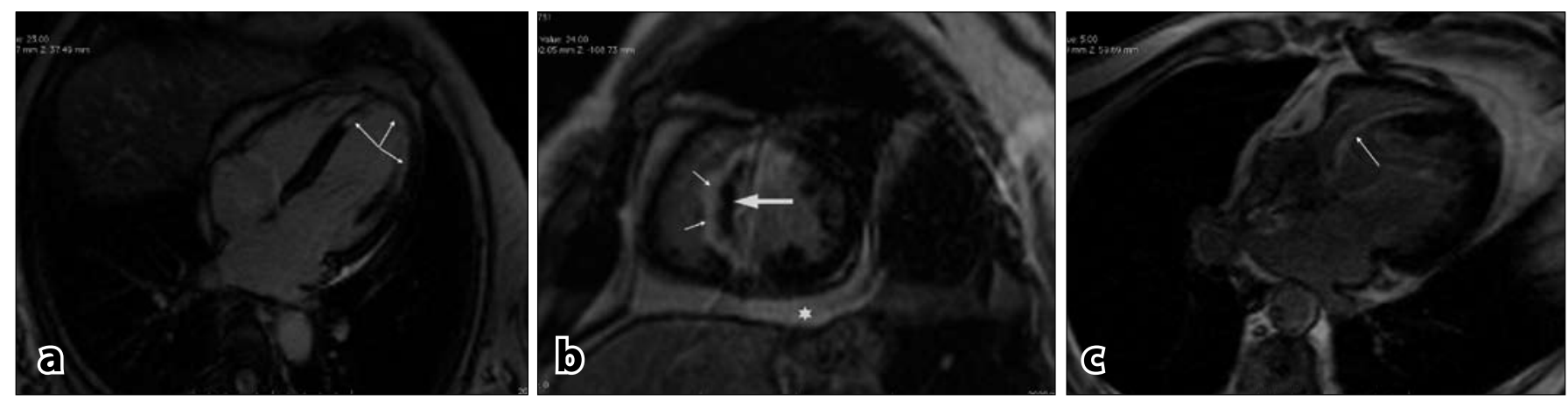

Fig. 7. Delayed enhancement images showing examples of infarction. (7a.) Subendocardial anteroseptal and apical LV infarction (arrows) on four-chamber orientation. (7b.) Acute transmural septal infarct (arrows) in short-axis orientation. The low signal intensity, subendocardial region within the infarct (block arrow) indicates 'no reflow' infarction (microvascular obstruction) associated with a poorer prognosis and increased chance of myocardial rupture in the acute setting and aneurysm formation in the chronic setting. Note the simple pericardial effusion (star). (7c.) Septal transmural infarct with aneurysm formation (arrow) on four-chamber orientation. 


\section{REVIEW ARTICLE}
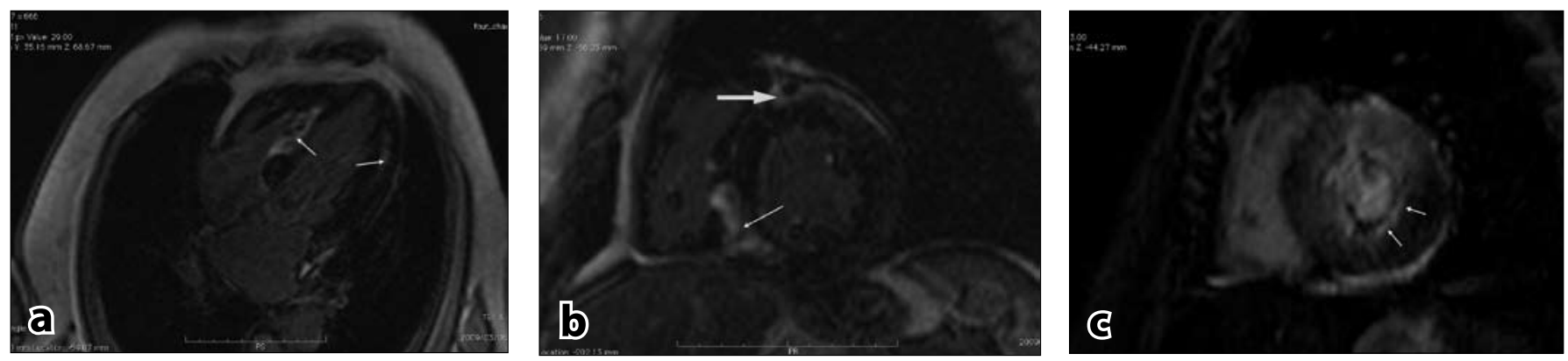

Fig. 8. Delayed enhancement CMR in non-ischaemic cardiomyopathies. (8a.) Four-chamber orientation showing multifocal enhancement (arrows) in a patient with cardiac sarcoidosis. (8b.) Short-axis orientation in cardiac sarcoidosis. The distribution of enhancement (subepicardial (block arrow) and at the LV-RV junction (arrow)) is typical. (8c.) Short axis image in cardiac amyloidosis. Note the inhomogeneous LV nulling indicating diffuse infiltration with typical distribution (subendocardial not limited to vascular territories) enhancement (arrows).

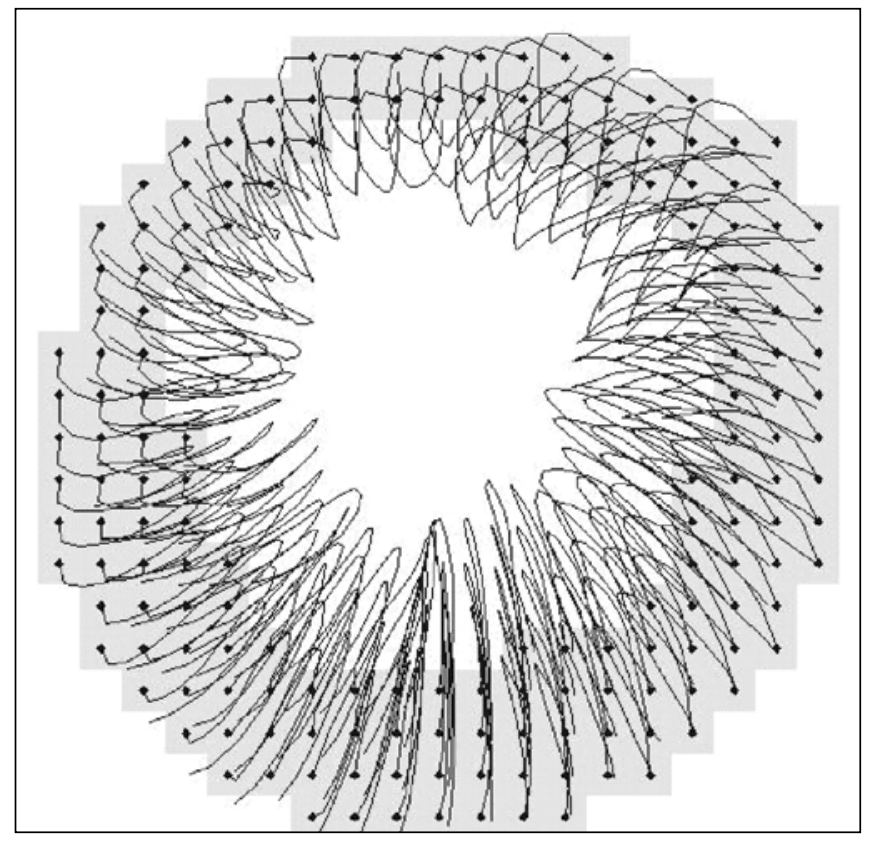

Fig. 9. Myocardial motion trajectories for a mid-ventricular short-axis view of a healthy volunteer. The septum is centred at 9 o'clock in the image.

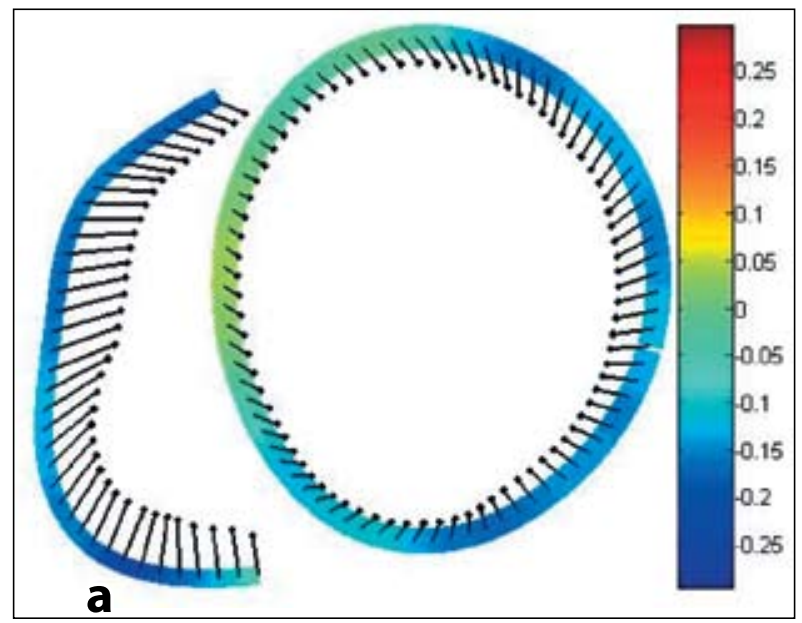

with congenital heart disease where intracardiac and extra-cardiac abnormalities often co-exist (e.g. tetralogy of Fallot with associated post-valvar pulmonary stenosis and aortic coarctation with associated VSD). Fig. 6 shows a post-contrast MR angiogram maximum intensity projection (MIP) image of a patient with Scimitar syndrome.

\section{Viability and delayed enhancement imaging}

MRI viability imaging is unparalleled by any other techniques. Gadolinium contrast agents settle in oedematous areas and take longer to be washed out of scar tissue. Delayed-enhancement MRI involves imaging the heart 10 minutes after administering the intravenous contrast using inversion recovery sequences that null normal myocardial tissue. Scar tissue appears unmistakeably bright in these images. This technique can map the severity and extent of myocardial infarction, with obvious prognostic implications (Fig. 7).

This technique is also increasingly being used to differentiate ischaemic from non-ischaemic cardiomyopathy. Typical patterns of delayed enhancement have been described for various non-ischaemic cardiomyopathies, ${ }^{9}$ allowing non-invasive diagnosis and prognostication (Fig. 8).

\section{Perfusion}

Myocardial perfusion can be quantified by rapidly measuring the uptake of gadolinium. A cine image series with manually introduced contours is

Fig. 10a. Mid-ventricular short-axis map of end-systolic tangential strain of a patient with a right bundle branch block (RBBB) and an anteroseptal infarct (arrows), and (10b) corresponding strain versus time curves. 
post-processed to yield regional measures of tissue perfusion. In contrast with the longer-term gadolinium uptake, ischaemic myocardium appears darker than normal myocardium in the first images owing to reduced perfusion. This can be incorporated into a stress test but requires the use of Adenosine, which is unfortunately not currently licensed for this application in South Africa.

\section{Coronary angiography}

Although MR coronary angiography has been steadily improving, this arguably remains the one shortcoming of CMR. Resolution lags behind multidetector CT, and techniques such as volume imaging with respiratory navigator tagging are not as robust.

\section{Myocardial tagging and DENSE}

In MRI tagging, the myocardium is modulated by a series of saturated dark bands or tags. These are a material property of the tissue and can be seen to deform as the heart moves. Tagged images provide insight into contractility and myocardial mechanics, and can be processed to yield meaningful measures of myocardial strain. ${ }^{10}$ Displacement encoding with stimulated echoes (DENSE) is a more recent technique that provides more accurate measures of displacement and hence myocardial strain. ${ }^{11,12}$ Both myocardial tagging and DENSE allow a user to track discrete portions of tissue as they traverse the cardiac cycle. Fig. 9 shows an example of this technique, where a number of points in the left ventricle are tracked through the cardiac cycle.

Myocardial dyssynchrony can be mapped by using tagging or DENSE regional strain-time curves. Dyssynchronous but healthy segments of myocardium can be identified using a variety of metrics such as the time to onset of strain or the time to peak strain. This technique can be used in combination with delayed-contrast MRI to improve the planning of cardiac resynchronisation therapy. Fig. 10 presents a patient with a right bundle branch block and an anteroseptal myocardial infarct. Fig. 10a is an end-systolic displacement and strain map obtained using cine DENSE imaging. The black lines represent motion relative to end-diastole, and the underlying colour represents tissue deformation corresponding to circumferential shortening. The infarct is clearly apparent as a region of zero or slightly positive strain. The positive strain occurs when infarcted tissue is stretched by the adjacent healthy tissue. Further information can be obtained by looking at regional strain as a function of time. The plots in Fig. 10b show strain for the anterior septum and regions in the right and left ventricular free walls. A delay in onset to contraction is clearly apparent in the RV segment, but the peak strain is normal; this implies that pacing the RV free wall would benefit this patient but pacing the anterior septum would yield no benefit.

\section{Conclusion}

Just as MRI has made and continues to make a major impact on neuroscience, sports medicine, orthopaedics and various other fields, it is currently also making a major impact in cardiology. CMR is established as the gold standard imaging technique for assessing myocardial and pericardial pathology. It is the most accurate and reproducible non-invasive investigation for ventricular function and viability assessment. CMR is also a valuable problem-solving adjunct to echocardiography in congenital and valvular heart disease. Inability to depict detailed coronary artery anatomy is the major shortcoming of CMR, but physicists continue to develop new and faster methods of imaging to overcome this factor and further improve established applications.

1. Haacke WM, Brown RW, Thompson MR, Venkatesan R. Magnetic Resonance Imaging: Physical Principles and Sequence Design. New York: John Wiley and Sons, 1999.

2. Blaimer M, Breuer F, Mueller M, Heidemann RM, Griswold MA, Jakob PM. SMASH, SENSE, PILS, GRAPPA: How to choose the optimal method. Top Magn Reson Imaging 2004;15:223-236.

3. Heidemann RM, Ozsarlak O, Parizel PM, et al. A brief review of parallel magnetic resonance imaging. Eur Radiol 2003;13:2323-2337.

4. Deborah CS, Geukes-Foppan M, Barry K, et al. Practical implications of liver and heart iron load assessment by T2*-MRI in children and adults with transfusion dependent anaemias. Am J Hematol 2008; 83(10):781-783.

5. Petersen SE, Jung BA, Wiesmann F, et al. Myocardial tissue phase mapping with cine phase-contrast MR imaging: Regional wall motion analysis in healthy volunteers. Radiology 2006;238(3):816-826.

6. Jung B, Föll D, Böttler P, Petersen S, Hennig J, Markl M. Detailed analysis of myocardial motion in volunteers and patients using high-temporal-resolution MR tissue phase mapping. J Magn Reson Imaging 2006;24:1033-1039.

7. Bock J, Wieben O, Johnson KM, Hennig J, Markl M. Optimal processing to derive static PC-MRA from time-resolved 3D PC-MRI data. Proc Intl Soc Mag Reson Med 2008;16:3053.

8. Anderson AG, Johnson KM, Bock J, Markl M, Wieben O. Comparison of image reconstruction algorithms for the depiction of vessel anatomy in PC VIPR datasets. Proc Intl Soc Mag Reson Med 2008;16:934.

9. Cummings KW, Bhalla S, Javidan-Nejad C, Bierhals AJ. A pattern-based approach to non-ischaemic cardiomyopathy at MR imaging. Radiographics 2009;1:89-103.

10. Axel L, Montillo A, Kim D. Tagged magnetic resonance imaging of the heart: a survey. Med Image Anal 2005;9:376-393.

11. Aletras AH, Ding S, Balaban RS, Wen H. DENSE: Displacement encoding with stimulated echoes in cardiac functional MRI. J Magn Reson 1999;137:247-252.

12. Kim D, Gilson WD, Kramer CM, Epstein FH. Myocardial tissue tracking with two-dimensional cine displacement-encoded MR imaging: Development and initial evaluation. Radiology 2004;230:862-871. 